\title{
Comparison of time-domain, spectral-domain and swept-source OCT in evaluating aqueous cells in vitro
}

\author{
Meng $\mathrm{Li}^{1}$, Huaizhou Wang ${ }^{1}$, Yue Liu ${ }^{2}$, Xifang Zhang ${ }^{1} \&$ Ningli Wang ${ }^{1 *}$ \\ ${ }^{1}$ Beijing Institute of Ophthalmology, Beijing Tongren Eye Center, Beijing Tongren Hospital, Capital Medical University; Beijing \\ Ophthalmology \& Visual Sciences Key Laboratory, Beijing 100005, China; \\ ${ }^{2}$ Department of Ophthalmology, The affiliated hospital of Guizhou Medical University, Guiyang 550001, China
}

Received May 31, 2016; accepted August 23, 2016; published online November 4, 2016

\begin{abstract}
Different optical coherence tomography (OCT), both time-domain OCT and spectral-domain OCT, has been used to evaluate anterior chamber cells in previous studies. Recently, commercial swept-source OCT was available. The comparison among three kinds of OCTs have not been done before. Here, we compared their feasibility in discerning aqueous cells in vitro. The peripheral blood was diluted to eight different cell concentrations and was packed into plastic-film bag. Each sample was tested using different scan modes of three OCTs. It was easy to discern aqueous cells from background noise in both Visante AS-OCT and RTVue XR Avanti OCT, but difficult in Casia SS-1000 OCT. The measured blood cell densities in high-resolution corneal cross-sectional scan of Visante AS-OCT, cornea line scan and 3D cornea scan of RTVue XR Avanti OCT were highly correlated with the actual blood cell concentration (Pearson correlation $=0.991,0.989$, and 0.993 respective-

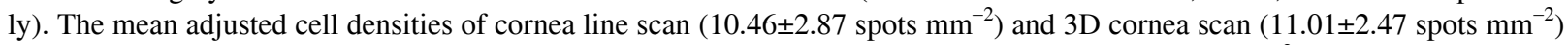
of RTVue XR Avanti OCT were significantly larger than that of Visante AS-OCT $\left(1.55 \pm 1.75\right.$ spots $\left.\mathrm{mm}^{-2}\right)(P<0.01)$. Our study demonstrated that RTVue XR Avanti OCT, a spectral-domain OCT, is superior to other two OCTs in evaluating aqueous cells.
\end{abstract}

optical coherence tomography, uveitis, aqueous cell

Citation: Li, M., Wang, H., Liu, Y., Zhang, X., and Wang, N. (2016). Comparison of time-domain, spectral-domain and swept-source OCT in evaluating aqueous cells in vitro. Sci China Life Sci 59, 1319-1323. doi: 10.1007/s11427-016-0145-x

\section{INTRODUCTION}

Aqueous cell is an important sign of anterior chamber inflammation (Jabs et al., 2005). There are several ways to quantify the aqueous cells, including slit-lamp biomicroscopy (Jabs et al., 2005), ultrasound biomicroscopy (UBM) (Peizeng et al., 2009), laser flare photometry (Ladas et al., 2005) and optical coherence tomography (OCT) (Agarwal et al., 2009; Igbre et al., 2014; Kumar et al., 2014; Li et al., 2013; Sharma et al., 2015).

Previous studies used OCT in grading anterior chamber inflammatory cells. These researches showed anterior segment OCT (AS-OCT) can detect anterior chamber inflam-

*Corresponding author (email: wningli@vip.163.com) matory cells in patients with uveitis (Agarwal et al., 2009; Igbre et al., 2014; Li et al., 2013; Sharma et al., 2015) or post ocular operation (Kumar et al., 2014). In addition, AS-OCT has also been used to quantitatively assess the anterior chamber cells in a small rodent model of uveitis (Pepple et al., 2016). Advantages of using OCT to evaluate anterior chamber cells are obvious. Firstly, it is a non-contact procedure compared to UBM (Peizeng et al., 2009). Secondly, it provides objective images compared to slit-lamp biomicroscopy (Kumar et al., 2014). Thirdly, it can be used in patients with corneal opacity compared to laser flare photometry (Ladas et al., 2005).

Time-domain OCT (TD-OCT) (Li et al., 2013) and spectral-domain OCT (SD-OCT) (Sharma et al., 2015) have been used in previous studies. Recently, swept-source OCT 
(SS-OCT) (Baskaran et al., 2013) was available. The comparison of different OCT in grading aqueous cells has not been studied before, thus we designed a research using an in-vitro model to compare and evaluate TD, SD and SS-OCT in discerning aqueous cells.

\section{RESULTS}

For Visante AS-OCT and RTVue XR Avanti OCT, the scattered background noise of the images was uniform. It was easy to discern the aqueous cells from the background noise in these two OCTs (Figure 1). However, the high background noise varied in Casia SS-1000 OCT, and the aqueous cells were mostly found in the anterior center (Figure 1). It is difficult to discern aqueous cells from the background noise in Casia SS-1000 OCT. So we only compared images obtained by Visante AS-OCT and RTVue XR Avanti OCT.

The blood cell density measured using high-resolution corneal cross-sectional scan of Visante AS-OCT was highly correlated with the actual blood cell concentration (Pearson correlation=0.991) (Figures 2 and 3). The linear regression provided an estimate of OCT-measured blood cell density,

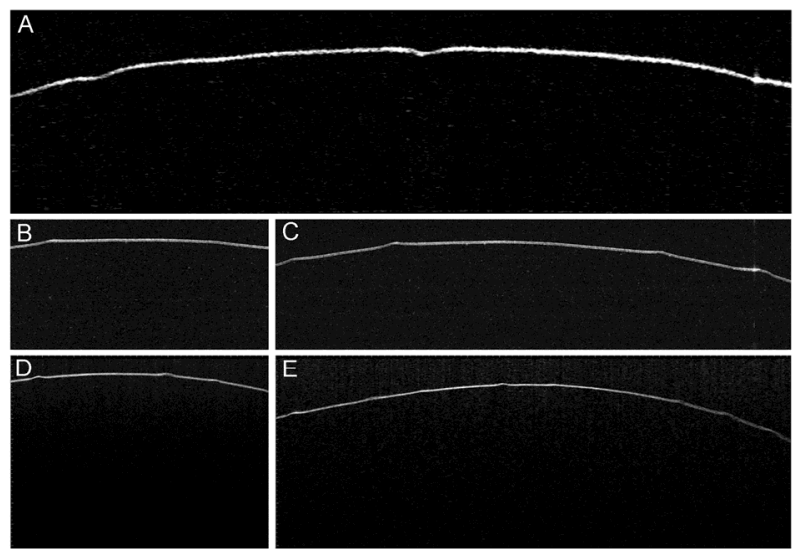

Figure 1 OCT images of different OCT scan modes for the same sample. A, High-resolution corneal cross-sectional scan of Visante AS-OCT. B, 3D cornea scan mode of RTVue XR Avanti OCT. C, Cornea line scan of RTVue XR Avanti OCT. D, Angle HD scan mode of Casia SS-1000 OCT. E, Anterior segment scan of Casia SS-1000 OCT. which equaled $0.0858 \times$ blood cell concentration -0.0419 $\left(R^{2}=0.982\right)$.

The blood cell density measured using cornea line scan of RTVue XR Avanti OCT was highly correlated with the actual blood cell concentration (Pearson correlation=0.989) (Figures 4 and 5). The linear regression provided an estimate of OCT-measured blood cell density, which equaled $0.5882 \times$ blood cell concentration $+0.2048\left(R^{2}=0.9774\right)$.

The blood cell density measured using $3 \mathrm{D}$ cornea scan of RTVue XR Avanti OCT was highly correlated with the actual blood cell concentration (Pearson correlation=0.993) (Figures 6 and 7). The linear regression provided an esti-
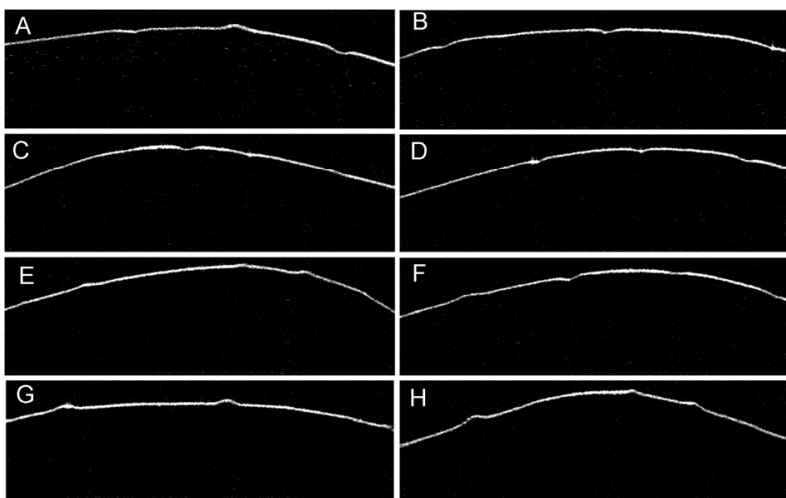

Figure 2 The Visante AS-OCT images for different samples. A-H represents sample A-H.

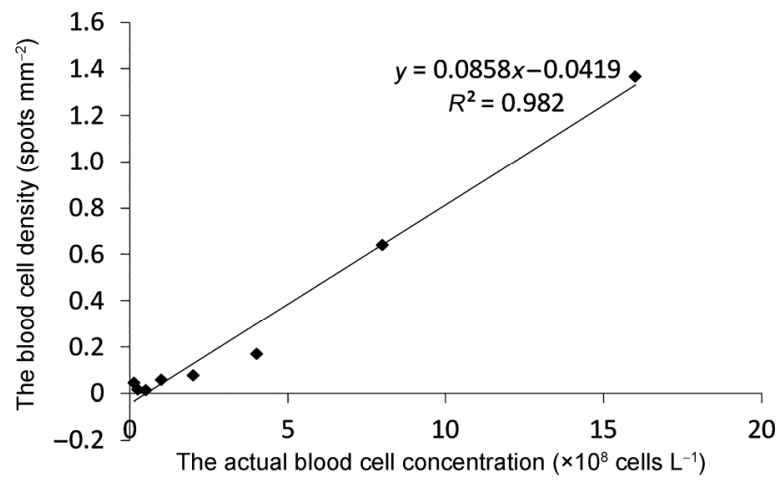

Figure 3 The blood cell density measured by Visante AS-OCT was highly correlated with the actual blood cell concentration.
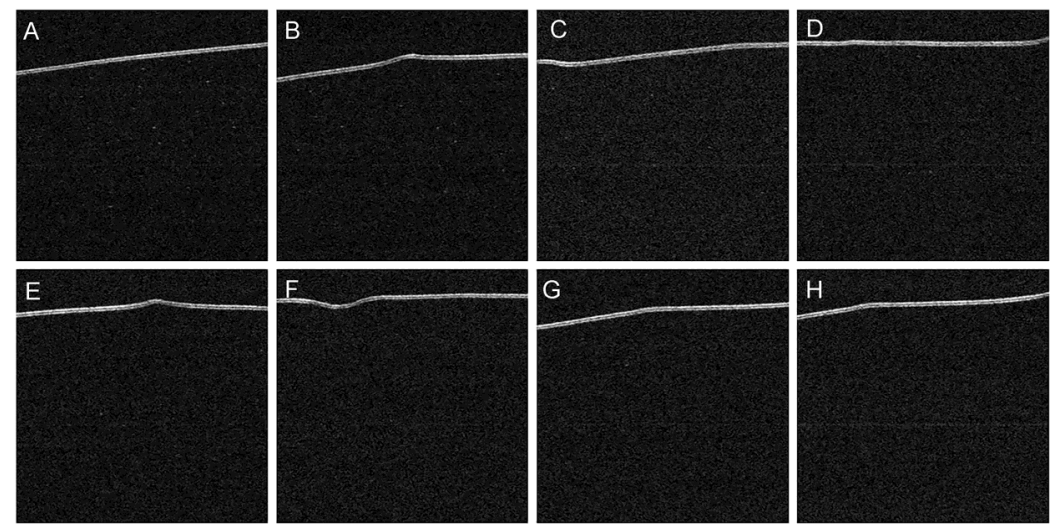

Figure 4 The cornea line scans of RTVue XR Avanti OCT for different samples. A-H represents sample A-H. 


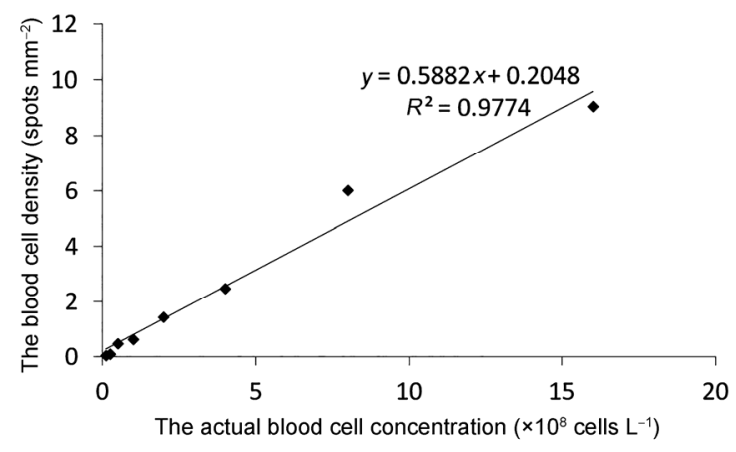

Figure 5 The blood cell density measured by cornea line scan of RTVue XR Avanti OCT was highly correlated with the actual blood cell concentration.

mate of OCT-measured blood cell density, which equaled $0.7676 \times$ blood cell concentration $+0.0454\left(R^{2}=0.9852\right)$.

The cell densities of eight samples measured by different OCT modes were listed in Table 1. The adjusted cell densities (mean \pm standard deviation) measured by high-resolution corneal cross-sectional scan of Visante AS-OCT, cornea line scan and 3D cornea scan of RTVue XR Avanti OCT were (1.55 \pm 1.75$),(10.46 \pm 2.87)$ and $(11.01 \pm 2.47)$ spots $\mathrm{mm}^{-2}$ respectively. One-way ANOVA showed significant difference among three OCT scan modes $(F=38.836, P<0.01)$. Further analysis with LSD- $t$ test revealed that the adjusted cell densities measured by cornea line scan and 3D cornea scan of RTVue XR Avanti OCT were not significantly different $(P=0.65)$, the adjusted cell densities measured by cornea line scan or 3D cornea scan of RTVue XR Avanti OCT were significantly larger than that of high-resolution corneal cross-sectional scan of Visante OCT $(P<0.01$, $P<0.01$ respectively).

The intrasession repeatability of measured aqueous cell density using different OCT scan modes was listed in Table 2. The intraclass correlation coefficient (ICC) of intrasession repeatability for high-resolution corneal cross-sectional scan of Visante AS-OCT, 3D cornea scan and cornea line scan of RTVue XR Avanti OCT was 0.975, 0.986 and 0.964 respectively.

\section{DISCUSSION}

We explored an in vitro model to discern aqueous cells using Visante AS-OCT, RTVue XR Avanti OCT and Casia SS-1000 OCT, and compared their ability to detect blood cells. Visante AS-OCT and RTVue XR Avanti OCT showed better performance than Casia SS-1000 OCT in discerning the aqueous cells. In addition, the adjusted cell densities measured by cornea line scan and 3D cornea scan of RTVue XR Avanti OCT were significantly larger than that of high-resolution corneal cross-sectional scan of Visante AS-OCT, suggesting that RTVue XR Avanti OCT
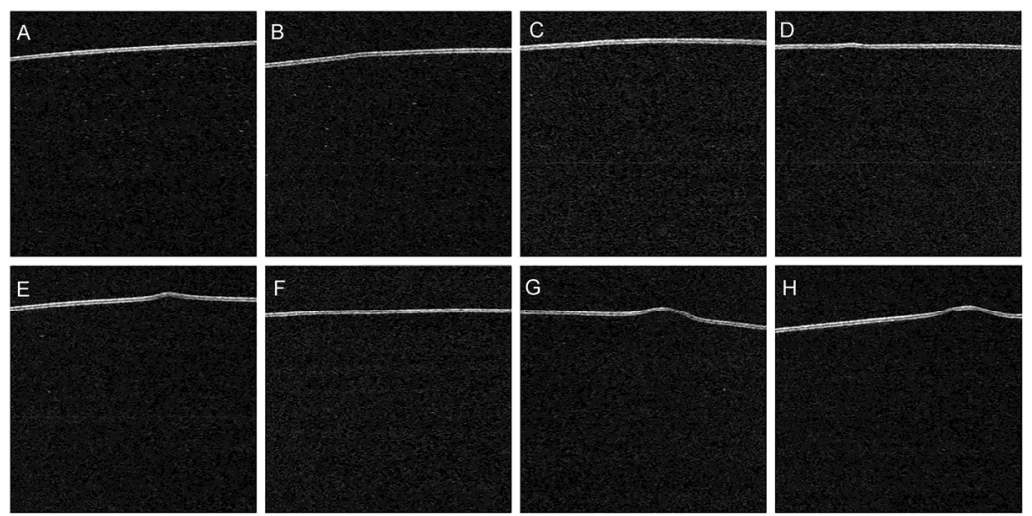

Figure 6 The 3D cornea scans of RTVue XR Avanti OCT for different samples. A-H represents sample A-H.

Table 1 The measured cell density and adjusted cell density of different OCT scan modes for eight samples

\begin{tabular}{|c|c|c|c|c|c|c|c|}
\hline \multirow{2}{*}{ Sample } & \multirow{2}{*}{$\begin{array}{l}\text { Actual cell concentration } \\
\qquad\left(\times 10^{8} \mathrm{~L}^{-1}\right)\end{array}$} & \multicolumn{3}{|c|}{ Measured cell density $\left(\right.$ spots $\mathrm{mm}^{-2}$ ) } & \multicolumn{3}{|c|}{ Adjusted cell density (spots $\mathrm{mm}^{-2}$ ) } \\
\hline & & Visante & RTVue cornea line & RTVue 3D cornea & Visante & RTVue cornea line & RTVue 3D cornea \\
\hline A & 16 & 1.368 & 9.071 & 11.8 & 1.368 & 9.071 & 11.8 \\
\hline B & 8 & 0.6437 & 6.041 & 7.385 & 1.2874 & 12.082 & 14.77 \\
\hline $\mathrm{C}$ & 4 & 0.1697 & 2.495 & 2.841 & 0.6788 & 9.98 & 11.364 \\
\hline $\mathrm{D}$ & 2 & 0.07986 & 1.459 & 1.715 & 0.63888 & 11.672 & 13.72 \\
\hline $\mathrm{E}$ & 1 & 0.05974 & 0.6528 & 0.5858 & 0.95584 & 10.4448 & 9.3728 \\
\hline $\mathrm{F}$ & 0.5 & 0.01609 & 0.4993 & 0.2759 & 0.51488 & 15.9776 & 8.8288 \\
\hline G & 0.25 & 0.01733 & 0.1132 & 0.1697 & 1.10912 & 7.2448 & 10.8608 \\
\hline $\mathrm{H}$ & 0.125 & 0.04542 & 0.05617 & 0.05766 & 5.81376 & 7.18976 & 7.38048 \\
\hline
\end{tabular}

Table 2 Intrasession repeatability of aqueous cell measurements in each sample using different OCT scan modes

\begin{tabular}{ccc}
\hline OCT scan mode & ICC & 95\% CI \\
\hline High-resolution corneal cross-sectional scan of Visante AS-OCT & 0.975 & $0.917-0.994$ \\
3D cornea scan mode of RTVue XR Avanti OCT & 0.986 & $0.952-0.997$ \\
Cornea line scan of RTVue XR Avanti OCT & 0.964 & $0.886-0.992$ \\
\hline
\end{tabular}




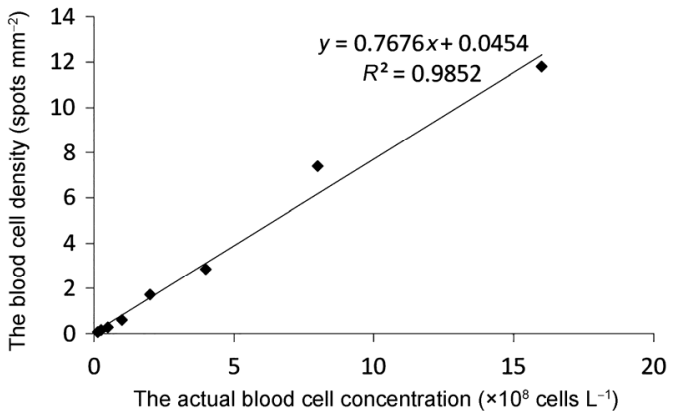

Figure 7 The blood cell density measured by 3D cornea scan of RTVue XR Avanti OCT was highly correlated with the actual blood cell concentration.

had higher resolution in discerning aqueous cells than Visante AS-OCT.

The measured cell densities of sample $G$ and $H$ were larger than that of sample F in Visante AS-OCT, which was not in concert with the actual cell concentration, which might originate from the lower resolution of Visante AS-OCT. Visante AS-OCT is not suggested to be used to quantify the aqueous cells when its concentration is lower than $5 \times 10^{7}$ cells $\mathrm{L}^{-1}$.

Many kinds of commercial OCTs were available in clinic to evaluate the anterior segment of eyeball (Agarwal et al., 2009; Igbre et al., 2014; Kumar et al., 2014; Li et al., 2013; Sharma et al., 2015). We only chose three commercial OCTs in this study, which represent three kinds of OCTs. To be specific, Visante AS-OCT is a TD-OCT with the lowest A-scan speed among these three OCTs. It was the first generation of OCT used to evaluate the aqueous cells. Several studies showed that it can quantify the aqueous cells both in vivo and in vitro (Agarwal et al., 2009; Igbre et al., 2014; Li et al., 2013). RTVue XR Avanti OCT is a SD-OCT with the highest A-scan speed among three OCTs we used which has never been employed in discerning aqueous cells. However, one study showed that RTVue-100 (Optovue Inc, USA) can be used to image and grade aqueous cells in vivo (Sharma et al., 2015), and clinical grading strongly correlates with the number of cells in OCT image. RTVue XR Avanti OCT is an upgraded version of RTVue-100, with higher A-scan speed. It employs the light source centered on $840 \mathrm{~nm}$. Cirrus HD Fourier-domain OCT (model 4000, Carl Zeiss Meditec AG) (Kumar et al., 2014), another SD-OCT, has also been used to evaluate the aqueous cells in vivo before. Previous studies showed that both TD-OCT and SD-OCT were reliable in assessing aqueous cells in patients with uveitis (Agarwal et al., 2009; Igbre et al., 2014; Li et al., 2013; Sharma et al., 2015) or post intraocular surgery (Kumar et al., 2014). Casia SS-1000 OCT is a swept-source anterior segment OCT, it was widely used in anterior segment imaging due to its high scan speed and large scan field (Baskaran et al., 2013). However, it was not used for discerning aqueous cells.

We used peripheral blood cells in our study, which consists of majorly red blood cells (RBCs). It was different from Li's study (Li et al., 2013), which used latex micro- sphere. The average diameter of latex microsphere was only $3 \mu \mathrm{m}$ ( $\mathrm{Li}$ et al., 2013). The cells in the anterior chamber in uveitis patients are mainly neutrophilis, lymphocytes, macrophages and monocytes (Chang et al., 2005; Rao, 2007). The average diameter of neutrophils ranges from 12 to $15 \mu \mathrm{m}$. The average diameter of lymphocytes is about $7 \mu \mathrm{m}$, and the average diameter of macrophage is about $21 \mu \mathrm{m}$. The average diameter of monocytes ranges from 7 to $10 \mu \mathrm{m}$. These four kinds of cells majorly in anterior chamber in uveitis are significantly larger than latex microsphere, but are similar to or slightly larger than peripheral red blood cell. Rose-Nussbaumer et al. compared the optical properties of three types of inflammation cells and RBCs using OCT. Their study showed that all cells appeared as hyperreflective spots in OCT images, and RBCs had similar average axial width with neutrophils, lymphocytes, and monocytes (Rose-Nussbaumer et al., 2015). Considering the comparable size of peripheral blood cells to inflammatory cells of uveitis patients, we chose peripheral blood cells as substitution in our study.

In our study, we evaluated the aqueous cells in vitro, comparing to known concentration peripheral blood, in order to test the feasibility of OCT on quantifying aqueous cells and to set a standard for further use in vivo. Slit-lamp biomicroscopy is currently the gold standard in grading anterior chamber inflammation (Jabs et al., 2005). The Standardization of Uveitis Nomenclature Working Group graded the anterior chamber cells on a scale of zero to four based on slit-lamp biomicroscopy (Jabs et al., 2005). However, it was a subjective and hemi-quantifying method to evaluate the anterior chamber inflammation. Several studies had evaluated the feasibility of OCT in grading anterior chamber cells in vivo and compared the results to silt-lamp biomicroscopy grading (Kumar et al., 2014; Sharma et al., 2015). However, there was no report on the OCT measurement in comparison with actual cell density. Using known concentration peripheral blood as a gold standard, we tested the accuracy of three OCTs in detecting aqueous cells and compared them quantitatively.

There were several limitations in this study. Firstly, we chose the peripheral blood cells, which was different from anterior chamber inflammation cells in vivo. Secondly, we only tested it in vitro. In practice, aqueous cells moves dynamically, which may affect OCT scan image, especially in OCT scan mode that required repeated scans. Thirdly, only three commercial OCTs were evaluated in our study.

Despite these limitations, our study demonstrated the feasibility of OCT to quantify the anterior chamber cells in vitro, and the results showed a strong correlation between the OCT measurement and actual cell concentration. OCT can be a good tool in monitoring anterior chamber inflammation, especially in the situation that the change of anterior chamber inflammation is difficult to be detected by slit-lamp microscopy. RTVue XR Avanti OCT, a SD-OCT, is superior to other two OCTs in evaluating anterior chamber cells. In conclusion, OCT system designed similar as RTVue XR Avanti OCT was recommended in quantifying the anterior chamber inflammation. 


\section{MATERIALS AND METHODS}

This study had prior approval from the Beijing Tongren Hospital Institutional Review Board, and followed the tenets of the Declaration of Helsinki. Written informed consent was obtained from healthy volunteer.

Peripheral blood was obtained from a healthy volunteer. As a criteria, the whole blood cell was counted by the automated hematology analyzer. Peripheral blood was anticoagulated with ethylene diamine tetraacetic acid. As listed below, the blood was diluted to eight different cell concentrations (Table 1) using $0.9 \% \mathrm{NaCl}$ solution. The cell concentration was $1.6 \times 10^{9}, 8 \times 10^{8}, 4 \times 10^{8}, 2 \times 10^{8}, 1 \times 10^{8}, 5 \times 10^{7}$, $2.5 \times 10^{7}$ and $1.25 \times 10^{7}$ cells $\mathrm{L}^{-1}$ in sample A-H.

Each sample was packed into a small thin plastic-film bag and scanned three times repetitively using three OCTs. The OCT we used in this study include Visante AS-OCT (Carl Zeiss Meditec, Inc., Ireland), RTVue XR Avanti with AngioVue OCT (Optovue Inc) and the Casia SS-1000 OCT (Tomey, Japan).

Visante AS-OCT is a TD-OCT. It utilizes light at 1,310 nm wavelength. The scanning speed is 2,000 axial scans per second. The axial resolution is $18 \mu \mathrm{m}$ and the lateral resolution is $60 \mu \mathrm{m}$. For each sample, more than three high-resolution corneal cross-sectional $(10 \mathrm{~mm} \times 3 \mathrm{~mm})$ scans were obtained.

RTVue XR Avanti with AngioVue OCT is a SD-OCT. It utilizes light at $840 \mathrm{~nm}$ wavelength. The scanning speed is $70,000 \mathrm{~A}$-scans per second. The axial resolution is $5 \mu \mathrm{m}$ and the beam width is $22 \mu \mathrm{m}$. The anterior segment optical adaptor lens was used for each scan. For each sample, two scan protocols were performed, including cornea line scan $(8 \mathrm{~mm} \times 2 \mathrm{~mm})$ and 3D cornea scan $(4 \mathrm{~mm} \times 2 \mathrm{~mm})$. More than 3 scans were obtained for each mode.

Casia SS-1000 OCT is a SS-OCT. The scanning speed is 30,000 A-scans per second. The axial resolution is less than $10 \mu \mathrm{m}$ and the lateral resolution is less than $30 \mu \mathrm{m}$. For each sample, two scan modes were performed, including anterior segment scan $(16 \mathrm{~mm} \times 6 \mathrm{~mm})$ and angle HD scan $(8 \mathrm{~mm} \times$ $6 \mathrm{~mm}$ ). More than three scans were obtained for each mode.

The hyperreflective spots seen in each OCT image were counted manually in a single masked fashion by one of the investigators (Xifang Zhang). The feasibility of discerning aqueous cells from background noise was also evaluated before cell counting for each OCT image. The area scanned in each OCT image was calculated using Image $\mathrm{J}$ software version 1.50 (National Institutes of Health). The cell density for each OCT image was calculated as the number of hyperreflective spots divided by the area in each OCT image.

\section{Statistical analysis}

Data analysis was conducted by SPSS software version 20.0 (SPSS Inc, USA). The correlation of OCT-measured cell density and the actual aqueous cell concentration was calculated using Pearson correlation. A positive correlation between the actual cell concentration and the cell density measured from the AS-OCT image was considered significant when $R \geq 0.5$ with $P<0.05$. The measured cell density of each OCT mode for eight samples did not follow normal distribution due to different dilution ratio (Table 1). The adjusted cell density was calculated by multiplying measured cell density by the dilution ratio (Table 1). For example, the adjusted cell density of sample B was calculated by multiplying measured cell density by two, and the adjusted cell density of sample $\mathrm{C}$ was calculated by multiplying measured cell density by four. One-Way ANOVA analysis was performed to determine whether the adjusted cell density among different OCT scan modes had significantly difference. Further analysis using LSD- $t$ test was performed to test the difference between every two modes. The intrasession correlation coefficient was calculated to test the repeatability of each OCT scan mode.

Compliance and ethics The author(s) declare that they have no conflict of interest.

\section{Acknowledgements This work was supported by Beijing Scholar Pro- gram}

Agarwal, A., Ashokkumar, D., Jacob, S., Agarwal, A., and Saravanan, Y. (2009). High-speed optical coherence tomography for imaging anterior chamber inflammatory reaction in uveitis: clinical correlation and grading. Am J Ophthalmol 147, 413-416.e413.

Baskaran, M., Ho, S.W., Tun, T.A., How, A.C., Perera, S.A., Friedman, D.S., and Aung, T. (2013). Assessment of circumferential angle-closure by the iris-trabecular contact index with swept-source optical coherence tomography. Ophthalmology 120, 2226-2231.

Chang, J.H., McCluskey, P.J., and Wakefield, D. (2005). Acute anterior uveitis and HLA-B27. Surv Ophthalmol 50, 364-388.

Igbre, A.O., Rico, M.C., and Garg, S.J. (2014). High-speed optical coherence tomography as a reliable adjuvant tool to grade ocular anterior chamber inflammation. Retina (Philadelphia, Pa.) 34, 504-508.

Jabs, D.A., Nussenblatt, R.B., and Rosenbaum, J.T. (2005). Standardization of uveitis nomenclature for reporting clinical data. Results of the First International Workshop. Am J Ophthalmol 140, 509-516.

Kumar, D.A., Agarwal, A., Sivangnanam, S., Ghorai, A., Murugan, B., Packiyalakshmi, S., and Chandrasekar, R. (2014). Aqueous reaction quantification after phacoemulsification: fourier-domain optical coherence tomography versus slitlamp biomicroscopy. J Cataract Refract Surg 40, 2082-2090.

Pepple, K.L., Choi, W.J., Wilson, L., Van Gelder, R.N., and Wang, R.K. (2016). Quantitative assessment of anterior segment inflammation in a rat model of uveitis using spectral-domain optical coherence tomography. Invest Ophthalmol Visual Sci 57, 3567-3575.

Ladas, J.G., Wheeler, N.C., Morhun, P.J., Rimmer, S.O., and Holland, G.N. (2005). Laser flare-cell photometry: methodology and clinical applications. Surv ophthalmol 50, 27-47.

Li, Y., Lowder, C., Zhang, X., and Huang, D. (2013). Anterior chamber cell grading by optical coherence tomography. Invest Ophthalmol Visual Sci 54, 258-265.

Peizeng, Y., Qianli, M., Xiangkun, H., Hongyan, Z., Li, W., and Kijlstra, A. (2009). Longitudinal study of anterior segment inflammation by ultrasound biomicroscopy in patients with acute anterior uveitis. Acta Ophthalmol 87, 211-215.

Rao, N.A. (2007). Pathology of Vogt-Koyanagi-Harada disease. Int Ophthalmol 27, 81-85.

Rose-Nussbaumer, J., Li, Y., Lin, P., Suhler, E., Asquith, M., Rosenbaum, J.T., and Huang, D. (2015). Aqueous cell differentiation in anterior uveitis using Fourier-domain optical coherence tomography. Invest Ophthalmol Visual Sci 56, 1430-1436.

Sharma, S., Lowder, C.Y., Vasanji, A., Baynes, K., Kaiser, P.K., and Srivastava, S.K. (2015). Automated analysis of anterior chamber inflammation by spectral-domain optical coherence tomography. Ophthalmology 122, 1464-1470.

Open Access This article is distributed under the terms of the Creative Commons Attribution License which permits any use, distribution, and reproduction in any medium, provided the original author(s) and source are credited. 